\title{
Land- and water-based exercises in rheumatoid arthritis patients: a series of case reports
}

\author{
Kim Nolte (DPhil) ${ }^{1}$ \\ Dina C Janse van Rensburg (MB ChB, MMed, MSc Sports Medicine) ${ }^{2}$ \\ Pieter E Krüger (DPhil) ${ }^{1}$ \\ ${ }^{1}$ Department of Biokinetics, Sport and Leisure Sciences, University of Pretoria, South Africa \\ ${ }^{2}$ Section Sports Medicine, University of Pretoria, South Africa
}

Correspondence to: Kim Nolte (kim.nolte@up.ac.za)

\begin{abstract}
Objective. To compare the effects of a 3-month land- and waterbased exercise programme among rheumatoid arthritis (RA) sufferers.

Methods. Patients with RA Functional Class I and II $(N=10)$ were randomly assigned to a land-based exercise group (Group $L$ ) $(N=4)$, water-based exercise group (Group W) $(N=4)$ or a control group (Group $C)(N=2)$. Testing parameters included swollen joint count (SJC), tender joint count (TJC), erythrocyte sedimentation rate $(\mathrm{ESR})$, haemoglobin $(\mathrm{Hb}), 50-\mathrm{ft}(15.2-\mathrm{m})$ walk test, grip strength, isokinetic strength of knee extensors and flexors, knee range of motion (ROM) and aerobic capacity.

Results. There were individual improvements in most of the physical status parameters tested for the experimental groups (land-based exercise group and water-based exercise group) while the general trend for the control group was that of deterioration. Appropriate land-based exercises did not appear to aggravate disease activity. However, the water-based exercise programme was superior in controlling the disease activity with regards to the tender and swollen joint counts.

Conclusion. Both exercise interventions appeared to be beneficial in the treatment of RA. Further research is required comparing various modes of exercises for the treatment of RA, using larger samples and evaluating the long-term effects.
\end{abstract}

\section{Introduction}

Arthritis is one of the main causes of human disability, limiting everyday activities such as dressing, climbing stairs, getting in and out of bed, or walking. ${ }^{1}$ Rheumatoid arthritis (RA) is the most common type of chronic inflammatory arthritis. ${ }^{2} \mathrm{RA}$ is characterised by inflammation of the synovial lining of the joints, which ultimately results in cartilage and bone destruction. ${ }^{3}$ RA can affect any joint, large or small; however, the small joints are the most commonly affected. Since RA is a systemic disease, other parts of the body may be involved in the inflammatory process. ${ }^{1}$

Although there is no cure for RA the condition can be managed with various strategies. ${ }^{4}$ The use of exercise in the treatment of patients with RA has been widely debated in the past. In the late 1800s, the concept of total bed-rest became the standard care. It was not until 1948 when the undesirable effects of prolonged bed-rest were described, that exercise resumed its role in arthritis therapy and rehabilitation. ${ }^{5}$
Over the past decades there has been growing evidence of the health benefits of physical activity for patients with RA. ${ }^{6}$

In RA various factors may lead to a decline in functional ability. Apart from the direct consequences of the disease on the function of joints and muscles, physical inactivity contributes further to stiffness of the joints, muscle weakness and cardiorespiratory deconditioning. ${ }^{7}$ Research shows that patients with RA are in general less fit and more at risk of comorbidities when compared with healthy, age-matched controls. ${ }^{8-10}$

The primary goal of exercise therapy for RA is to improve joint mobility, muscle strength and aerobic and functional capacity. ${ }^{11}$ However, there is a debate as to what type of exercise would be the best for RA patients. ${ }^{5}$ Hydrotherapy has been shown to increase muscle strength, increase joint range of motion, improve aerobic capacity, reduce pain and improve function. ${ }^{12}$ The buoyancy of water and the ability to control its temperature make it favourable for patients with muscular and joint disease. Although most research conducted suggests that exercises in water are beneficial for RA patients, numerous problems exist with the prescription of water therapy programmes. For example, proper water facilities for exercise therapy are not always available. Heated pools designed for exercise therapy are expensive and maintenance is also time consuming and costly.

Home exercise programmes, usually consisting of land-based exercise, are often prescribed for RA patients. Land-based exercises, specifically weight-bearing exercise, also have the advantage of strengthening the connective tissue surrounding the joints and stimulating bone formation. ${ }^{5}$ These qualities are desirable because of the well-known complications of accelerated generalised osteoporosis induced by active inflammation, immobility and medication (cortisone) in RA. ${ }^{11}$

In the past much research on RA patients and exercise has focused on water-based exercises. Although recently more research has started focusing on land-based exercises, several questions remain unanswered. ${ }^{13}$ The efficacy of land-based exercise intervention with respect to pain, disease activity, functional ability, quality of life and structural damage remain unclear. ${ }^{14}$ Therefore, the aim of this study was to determine the outcome of exercise therapy, specifically comparing the effects of a land-based exercise programme with that of a water-based exercise programme in RA sufferers. 
TABLE I. Gender, age and anthropometry

\begin{tabular}{lcccc}
\hline Subject & Gender & Age (years) & Stature (cm) & Body mass (kg) \\
\hline W1 & Female & 66 & 159.1 & 92.0 \\
W2 & Female & 64 & 161.7 & 85.8 \\
W3 & Female & 52 & 161.4 & 71.0 \\
W4 & Female & 52 & 162.7 & 68.1 \\
L1 & Male & 60 & 199.6 & 105.4 \\
L2 & Female & 41 & 168.8 & 52.1 \\
L3 & Female & 57 & 170.4 & 97.3 \\
L4 & Female & 53 & 169.2 & 60.2 \\
C1 & Female & 43 & 167.4 & 54.6 \\
C2 & Female & 53 & 170.0 & 60.0 \\
average & & $54.1 \pm 8.1$ & $169.0 \pm 11.5$ & $74.7 \pm 9.1$ \\
\multicolumn{2}{l}{ W $=$ water-based exercise programme; = land-based exercise programme; C control. } & &
\end{tabular}

TABLE II. Total tender and swollen joint counts

\begin{tabular}{|c|c|c|c|c|c|c|c|c|}
\hline \multirow[b]{2}{*}{ Subject } & \multicolumn{4}{|c|}{ Total tender joint count } & \multicolumn{4}{|c|}{ Total swollen joint count } \\
\hline & Pre & Post & Difference & $\begin{array}{c}\text { Clinically } \\
\text { relevant }\end{array}$ & Pre & Post & Difference & $\begin{array}{l}\text { Clinically } \\
\text { relevant }\end{array}$ \\
\hline W1 & 6 & 2 & -4 & Yes & 5 & 1 & -4 & Yes \\
\hline W2 & 24 & 10 & -14 & Yes & 14 & 10 & -4 & Yes \\
\hline W3 & 11 & 8 & -3 & No & 12 & 8 & -4 & Yes \\
\hline W4 & 10 & 6 & -4 & Yes & 4 & 6 & 2 & No \\
\hline L1 & 11 & 15 & 4 & Yes & 13 & 13 & 0 & No \\
\hline L2 & 9 & 6 & -3 & No & 9 & 8 & -1 & No \\
\hline L3 & 10 & 10 & 0 & No & 10 & 9 & -1 & No \\
\hline L4 & 13 & 10 & -3 & No & 13 & 11 & -2 & No \\
\hline C1 & 8 & 8 & 0 & No & 10 & 10 & 0 & No \\
\hline C2 & 19 & 19 & 0 & No & 16 & 16 & 0 & No \\
\hline
\end{tabular}

\section{Methods}

Ethical clearance for this study was obtained from the Faculty of Humanities research proposal and ethics committee of the University of Pretoria, South Africa. All subjects were required voluntarily to read and sign an informed consent.

\section{Subjects}

Patients with classical or definite RA and mild to moderate disease activity (American College of Rheumatology Functional Class and II), were randomly assigned to the land-based exercise group (Group L), water-based exercise group (Group W) or non-exercise group (Group C). All patients were on stable medication. Exclusion criteria included the presence of unstable cardiopulmonary disease, acute joint symptoms and current participation in a physical fitness programme or organised sports activity.

\section{Exercise intervention}

Subjects in the exercise groups were required to exercise 2 - 3 times per week for a 3-month period. Those in the control group were instructed to continue with their normal sedentary lifestyle. Group L and $\mathrm{W}$ attended their rehabilitation sessions at the University of Pre- toria rehabilitation gymnasium and hydrotherapy pool, respectively. The same biokineticist instructed both groups throughout their participation in the study.

Both the land- and water-based exercise programmes were aimed at improving range-of-motion, muscle strength and cardiorespiratory endurance. The exercise intervention consisted of warm-up exercises, strengthening exercises, aerobic exercises and cool-down exercises with stretches. Initially, the duration of the warm-up and strengthening phases was longer in order to build muscle strength. Aerobic exercise time was gradually increased as cardiorespiratory fitness improved. The total duration of an exercise session in each of the 2 programmes was approximately 45 minutes each.

\section{Assessments}

Each patient was assessed 3 times throughout the study to track progress: before the exercise intervention, 5 - 6 weeks into the exercise intervention and at the end of the exercise intervention (3 months). The results of only the pre- and post-exercise assessments are reported. 


\section{Clinical assessment}

A medical specialist rheumatologist performed all clinical assessments. Joints were examined for soft-tissue swelling and tenderness and pain during motion, using the American College of Rheumatology, rheumatoid arthritis clinical response criteria. ${ }^{15}$

\section{Haematological assessment}

Blood samples were drawn and standard laboratory procedures were used to estimate erythrocyte sedimentation rate (ESR) (modified Westergren, $\mathrm{mm} / \mathrm{h})$ and haemoglobin $(\mathrm{Hb})(\mathrm{gm} / \mathrm{dl}){ }^{16}$

\section{Physical status assessment}

The following functional assessments were performed:

- A 50-ft (15.2m) walk test. $^{3}$

- Manual grip strength was measured with a sphygmomanometer cuff rolled up two turns and inflated to $20 \mathrm{mmHg}$.

- The strength of the knee extensors and flexors was tested on an isokinetic dynamometer (Cybex Norm 7000). A speed of $60 \%$ second was used, ${ }^{3}$ trial repetitions and 5 test repetitions were performed.

- Knee range of motion was measured using a standard goniometer.

- Bicycle ergometer testing was performed to determine aerobic capacity. The Astrand-Rhyming protocol to obtain data for calculating the estimated $\mathrm{VO}_{2 \text { max }}$ of each subject was used. ${ }^{3}$ The test was started with an initial load of 25 watts (W) at a cadence of 60 - 70 revolutions per minute with an increment increase of $25 \mathrm{~W}$ until exhaustion.

\section{Statistical analyses}

Computations to determine standard descriptive statistics (mean and standard deviation) for age, stature and body mass of participants were performed using the Statistical Package for Social Sciences (SPSS), Microsoft Windows release 9.0 (1999). Due to the small sample the raw data and the difference between the pre and post data are presented for each subject. These changes are interpreted in the context of clinically meaningful results for each variable.

\section{Results}

Ten patients ( 9 females, 1 male) with classic or definite RA volunteered for the study. The mean $( \pm S D)$ age, stature, and body mass of the subjects was $54.1 \pm 8.1$ years, $169.0 \pm 11.5 \mathrm{~cm}$ and $74.7 \pm 9.1 \mathrm{~kg}$, respectively. Subjects were randomly assigned to Group L, W or C (Table I).

\section{Clinical and haematological assessment results}

An increase or decrease of 4 joints may be considered to be clinically significant or a meaningful change for the joint counts. The total TJC decreased in all the subjects in group W. The decreases were all deemed to be clinically significant. In group L, the total TJC decreased in two of the subjects, remained unchanged in one and increased in another. None of the changes was deemed to be clinically relevant except for the subject whose total TJC increased. There was no change in the total TJC of the subjects in group $C$. The total SJC decreased in a clinically meaningful way in 3 of the 4 subjects in group W. One subject's SJC increased in group W; however, the increase was not deemed to be clinically relevant. There was a nonclinically relevant decrease in 3 of the subjects' SJC in group $L$ and 1 subject's SJC was unchanged. There was no change in the SJC of the subjects in group $\mathrm{C}$ (Table II). The haemoglobin values remained

\section{TABLE III. Erythrocyte sedimentation rate}

Erythrocyte sedimentation rate $\left(\mathrm{mm} \cdot \mathrm{h}^{-1}\right)$

\begin{tabular}{lcccc} 
Subject & Pre & Post & Difference & Within clinical reference range \\
\hline W1 & 10 & 20 & 10 & Yes (pre and post) \\
W2 & 20 & 10 & -10 & Yes (pre and post) \\
W3 & 50 & 26 & -24 & No (pre) Yes (post) \\
W4 & 2 & 2 & 0 & Yes (pre and post) \\
L1 & 7 & 10 & 3 & Yes (pre and post) \\
L2 & 9 & 7 & -2 & Yes (pre and post) \\
L3 & 30 & 42 & 12 & Yes (pre) No (post) \\
L4 & 13 & 7 & -6 & Yes (pre and post) \\
C1 & 18 & 18 & 0 & Yes (pre and post) \\
C2 & 30 & 14 & -16 & Yes (pre and post)
\end{tabular}

$\mathrm{W}=$ water-based exercise programme; $\mathrm{L}=$ land-based exercise programme; $\mathrm{C}=$ control.

unchanged in all 3 groups. There were changes in the ESR in the groups; however, no specific trends were identified and values generally fell within normal clinical reference ranges (Table III).

\section{Physical status assessments results}

Various aspects of physical conditioning are shown in Tables IV and V. There was an improvement in group $\mathrm{W}$ and group L's physical condition as determined by the 50-ft (15.2-m) walk and aerobic capacity test for all subjects, while there were no improvements noted in group C. In general, for other variables there were trends of improvement for group $\mathrm{W}$ and group $\mathrm{L}$ but not group $\mathrm{C}$.

\section{Discussion}

There is a growing interest among health professionals in improving the care of patients afflicted with chronic disabling diseases such as RA. In particular there is interest on the effects of exercise training programmes on the measurements of improvement in joint function, mobility, strength, endurance and cardiovascular fitness. ${ }^{17}$ One of the aims of this study was to determine whether exercise therapy is beneficial for RA patients. The positive changes produced by the land- and water-based exercise programmes are evident in the results as far as the disease (total and swollen joint count) and physical status of the subjects were concerned.

The exercise therapies appeared to assist in the control of the disease activity as both the TJC and SJC were reduced in most of the subjects in the experimental groups, but not in the control group. There was a decrease in 6 of the subject's joint counts (TJC and SJC) in the experimental groups, 3 of which were clinically significant decreases. No specific trends could be identified in the ESR. However, in the experimental groups, 3 subjects' ESR increased, 1 in group $W$ and 2 in group L. Despite the increases, values fell within acceptable ranges with the exception of 1 subject in group L. However, this subject's ESR was on the border of the acceptable range even before the start of the intervention. In the control group 1 subject's ESR remained unchanged and the other subjects decreased. It is important to note that ESR can be influenced by factors other than RA, such as anaemia, pregnancy and age. Therefore it may be useful to conduct other haematological assessments in addition to ESR such as C-reactive protein (CRP) to get a true reflection of the inflammatory status of the disease. 
TABLE IV. 50 -ft $(15.2 \mathrm{~m})$ walk test and relative maximal oxygen consumption

\begin{tabular}{|c|c|c|c|c|c|c|}
\hline \multirow[b]{2}{*}{ Subject } & \multicolumn{3}{|c|}{50 -ft walk test (s) } & \multicolumn{3}{|c|}{ Maximal oxygen consumption $\left(\mathrm{ml}^{\mathrm{kgg}}{ }^{-1} \cdot \mathrm{min}^{-1}\right)$} \\
\hline & Pre & Post & Difference & Pre & Post & Difference \\
\hline W1 & 10.1 & 9.1 & -1.0 & 11.6 & 19.7 & 8.1 \\
\hline W2 & 10.5 & 7.5 & -3.0 & 20.7 & 23.9 & 3.2 \\
\hline W3 & 11.4 & 9.7 & -1.7 & 21.9 & 35.9 & 14 \\
\hline W4 & 9.6 & 8.0 & -1.6 & 36.4 & 38.6 & 2.2 \\
\hline L1 & 8.0 & 6.8 & -1.2 & 17.2 & 22.3 & 5.1 \\
\hline L2 & 8.3 & 6.9 & -1.4 & 37.8 & 41.0 & 3.2 \\
\hline L3 & 9.2 & 7.8 & -1.4 & 15.8 & 23.2 & 7.4 \\
\hline L4 & 8.0 & 6.9 & -1.1 & 45.2 & 48.8 & 3.6 \\
\hline C1 & 7.5 & 7.3 & -0.2 & 38.1 & 30.6 & -7.5 \\
\hline $\mathrm{C} 2$ & 9.4 & 9.5 & 0.1 & 32.0 & 32.1 & 0.1 \\
\hline
\end{tabular}

\section{TABLE V. Physical parameters}

\begin{tabular}{|c|c|c|c|c|c|c|c|c|c|c|}
\hline \multirow[t]{2}{*}{ Subject } & \multicolumn{2}{|c|}{ Grip (mmHg) } & \multicolumn{2}{|c|}{$\begin{array}{l}\text { Isokinetic flexion } \\
(\mathrm{Nm} / \mathrm{kg})\end{array}$} & \multicolumn{2}{|c|}{$\begin{array}{c}\text { Isokinetic extension } \\
(\mathrm{Nm} / \mathrm{kg})\end{array}$} & \multicolumn{2}{|c|}{$\begin{array}{c}\text { Range of motion }\left({ }^{\circ}\right) \\
\text { flexion }\end{array}$} & \multicolumn{2}{|c|}{$\begin{array}{c}\text { Range of motion }\left({ }^{\circ}\right) \\
\text { extension }\end{array}$} \\
\hline & $\mathrm{L}$ & $\mathrm{R}$ & $\mathrm{L}$ & $\mathrm{R}$ & $L$ & $\mathrm{R}$ & $L$ & $\mathrm{R}$ & $L$ & $\mathrm{R}$ \\
\hline W1 & 138 & 162 & 29.3 & 21.7 & 64.1 & 64.1 & 84 & 79 & 0 & 0 \\
\hline W2 & 100 & 103 & 8.2 & 9.4 & 40.0 & 49.4 & 105 & 115 & 0 & 0 \\
\hline W3 & 80 & 102 & 65.0 & 66.2 & 125.4 & 140.8 & 130 & 125 & 0 & 0 \\
\hline W4 & 108 & 120 & 50.0 & 39.7 & 70.6 & 66.2 & 121 & 121 & 0 & 0 \\
\hline L1 & 265 & 265 & 83.8 & 64.8 & 168.6 & 140.0 & 120 & 125 & 9 & 14 \\
\hline L2 & 60 & 62 & 84.1 & 98.1 & 203.8 & 238.0 & 125 & 124 & 0 & 0 \\
\hline L3 & 81 & 85 & 47.4 & 48.5 & 101.0 & 89.7 & 112 & 115 & 0 & 0 \\
\hline L4 & 91 & 90 & 68.3 & 85.0 & 116.7 & 141.7 & 130 & 115 & 0 & 0 \\
\hline $\mathrm{C} 1$ & 59 & 41 & 92.6 & 85.2 & 175.9 & 188.9 & 131 & 130 & 0 & 0 \\
\hline $\mathrm{C} 2$ & 44 & 40 & 55.0 & 65.0 & 128.3 & 151.7 & 122 & 125 & 0 & 0 \\
\hline
\end{tabular}

Most of the physical status parameters assessed were positively influenced by both exercise therapies. The improvements in aerobic capacity are especially noteworthy due to the fact that in the past exercise therapy in RA primarily aimed at maintaining joint mobility and muscle strength. ${ }^{14}$ However, because of the increased risk of cardiovascular events such as atherosclerosis of the coronary artery, aerobic training and fitness should be given the sufficient attention it deserves with regards to exercise programming. Other compelling evidence advocating the importance of aerobic exercises for RA sufferers was that of a systematic review and meta-analysis by Baillet and colleagues. ${ }^{14}$ The study found that aerobic exercises improve some of the most important RA patient outcomes: function, quality of life and pain. Moreover, it appears that aerobic exercise decreases radiological damage and pain. ${ }^{14} \mathrm{VO}_{2 \mathrm{max}}$ or $\mathrm{VO}_{2 \text { peak }}$ is considered a measure for aerobic fitness. In this study both the land- and waterbased exercise groups similarly improved their relative and absolute $\mathrm{VO}_{2 m a x}$ as well as their 50 -ft walk test time (Table IV). Another study has shown that $\mathrm{VO}_{2 \max }$ of subjects improved by $12 \%$ after following a 12-week endurance training programme. ${ }^{18}$ In contrast, in the nonexercise group there were no improvements in aerobic capacity parameters tested. In fact, one of the subjects showed deterioration. It is important to note that even small changes can be expected to have a detrimental impact on a RA patient, especially if one considers that the decline took place over a short period of time.

Due to the involvement of the joints of the hand and wrist, RA can influence grip strength. Therefore grip strength is considered an important measurement of a RA patient's functional status. Poor grip strength can affect activities of daily living such as the ability to open and close small buttons, to write and to perform any function related to work or housework. ${ }^{19}$ The exercise therapies appeared to have a positive effect on grip strength in comparison to the control group. In the experimental groups, 7 of the subjects' left grip strength improved and 4 of the subjects' right grip strength improved. In the control group there was a decrease in both left and right grip strength for both subjects except for 1 subject whose right grip strength minimally improved (Table V).

Impaired muscle function is a common consequence in patients with RA. ${ }^{8}$ It is important to maintain normal muscle strength, not only to maintain physical function, but also to stabilise the joints and prevent joint angulation and later osteoarthrosis. ${ }^{16}$ In general, 
there was an increase in knee extensor and flexor strength in the experimental groups and a deterioration in the control group. There appeared to be a more consistent improvement in the knee flexor strength in relation to knee extensor strength in the experimental groups. This may be due to the fact that there were muscle strength imbalances between the knee extensors and flexors at the start of the intervention (Table V).

The maintenance of functional ROM is necessary for daily activity and efficiency of movement. There were no significant deviations in knee extension ROM in relation to normative data before the exercise interventions and therefore no large changes were expected. Knee flexion ROM did however improve for all the subjects in both experimental groups and stayed approximately the same for the subjects in the control group (Table V).

It appears that exercise therapy does indeed play an important role in the treatment of rheumatic disease and in the fight against rheumatic invalidism. However, the primary purpose of the study was to determine which exercise mode, water-based exercise therapy or land-based exercise therapy, would be more effective in the treatment of RA. Importantly, results indicate that disease activity was not exacerbated by the land-based exercises. It is however worthwhile noting that there were more clinically significant decreases in the joint counts for the subjects following water-based therapy than landbased therapy. In addition, the TJC increased in one of the subjects following land-based therapy. The greater reduction in joint swelling and tenderness in the water-based exercise group may be attributed to the reduction of joint loading occasioned by the buoyancy. In addition, the hydrostatic pressure of water immersion is considered to reduce oedema. ${ }^{20}$

Concerning the physical status of the subjects in the experimental groups, both exercise therapies had a positive influence on most of the physical status parameters measured. However, it would appear that the improvements in the water-based exercise group seemed slightly more substantial than those of the land-based exercise group.

\section{Conclusion}

The results indicate that the benefits derived from both land- and water-based exercises are very similar. The prescription of landbased exercises is feasible, especially when hydrotherapy is not possible or contraindicated. The appropriate land-based exercises do not appear to enhance disease activity. Therefore the importance and unique benefits of land-based exercises should be considered when prescribing exercises for RA patients. It is possible that the exercise of choice for RA patients should not be water-based exercises alone, as believed in the past, but an optimal combination of landand water-based exercises. Ideally, the contribution of land- and water-based exercises to the overall programme of the RA patient should be manipulated according to the patient's needs and disease activity at that period of time.

Finally, it is important to emphasise the fact that this was a preliminary study and therefore the sample was small and any changes in disease symptoms must be viewed in the context of the natural course of RA, where daily fluctuations in joint motion, swelling, pain and tenderness are not uncommon. However, all patients were on stable medication, thus eliminating the possibility of confounding results due to changes in medication. Thus the results from this study indicate that future research focusing on the effects of various exercise modalities as well as the long-term effects of exercise interventions in the treatment of RA patients could prove valuable.

\section{REFERENCES}

1. Nieman DC. Exercise Soothes Arthritis. Joint effects. ACSMS Health Fit J 2000;4(3):20-27.

2. Thompson JM. Arthritis: Everything you need to know about arthritis. South Africa: Zebra Press, 1998:23-46.

3. Norceau L, Martineau H, Ro L, Belzile M. Effects of a modified dance based exercise on cardiorespiratory fitness, psychological state and health status of persons with Rheumatoid Arthritis. Am J Phys Med Rehab 1995;74(1):19-27.

4. Giannini MJ, Protas EJ. Exercise response in children with and without juvenile rheumatoid arthritis: A case comparison study. Phys Ther 1992;72(5):365-372.

5. Kirsteins AE, Dietz FD, Hwang SW. Evaluating the safety and potential use of a weight-bearing exercise, Tai-Chi Chuan, for rheumatoid arthritis patients. Am J Phys Med Rehab 1991;70(3):136-141.

6. Gaudin P, Leguen-Guegan S, Allenet B, Baillet A, Grange L, Juvin R. Is dynamic exercise beneficial in patients with rheumatoid arthritis? Joint Bone Spine 2008;75:11-17.

7. Van den Ende CHM, Hazes JMW, le Cessie S, et al. Comparison of high and low intensity training in well controlled rheumatoid arthritis. Results of a randomized clinical trial. Ann Rheum Dis 1996;55:798-805.

8. Ekdahl C, Broman G. Muscle strength, endurance, and aerobic capacity in rheumatoid arthritis: a comparative study with healthy subjects. Ann Rheum Dis 1992:51:35-40.

9. del Rincon ID, Williams K, Stern MP, Freeman GL, Escalante A. High incidence of cardiovascular events in a rheumatoid arthritis cohort not explained by traditional cardiac risk factors. Arthritis Rheum 2001;44:27372745.

10. Bacon PA, Townend JN. Nails in the coffin: increasing evidence for the role of rheumatic disease in the cardiovascular mortality of rheumatoid arthritis. Arthritis Rheum 2001;44:2707-2710.

11. Hazes JMW, Van den Ende CHM. How vigorously should we exercise our rheumatoid arthritis patients? Ann Rheum Dis 1996;55:861-862.

12. Tork SC, Douglas V. Arthritis water exercise program evaluation. A selfassessment survey. Arthrit Care Res 1989;2(1):28-30.

13. Klepper SE. Effects of an eight-week physical conditioning programme on disease signs and symptoms in children with chronic arthritis. Arthrit Care Res 1999;12(1):52-60.

14. Baillet A, Zeboulon N, Gossec L, et al. Efficacy of cardiorespiratory aerobic exercise in rheumatoid arthritis: Meta-analysis of randomized controlled trials. Arthrit Care Res 2010;62(7):984-992.

15. Ritchie DM, Doyle JA, McGinnis JM. Clinical studies with an articular index for the assessment of joint tenderness in patients with RA. Q J Med 1968:37:393-406.

16. Lyngberg KK, Ramsing BU, Nawracki A, Harreby M. Safe and effective knee extension training in Rheumatoid Arthritis. Arthritis \& Rheum 1994;37(5):623-628.

17. Komatireddy GR, Leitch RW, Cella K, Browning G, Minor M. Efficacy of low load resistive muscle training in patients with rheumatoid arthritis functional class II and III. J Rheumatol 1997;24(8):1531-1539.

18. Janse van Rensburg DC, Viljoen M, Coertzen C, et al. Efficacy of an exercise programme on the functional capacity and disease activity in females with rheumatoid arthritis. SAOJ 2010;28:685-691.

19. Minor MA, Hewett, JE. Physical fitness and work capacity in women with rheumatoid arthritis. Arthrit Care Res 1995;8(3):146-154.

20. Hall J, Maddison PJ, Chapman K. Arthrit Care Res 2010;9(3):206-215. 